PROCEEDINGS OF THE

AMERICAN MATHEMATICAL SOCIETY

Volume 132, Number 3, Pages 853-855

S 0002-9939(03)07137-5

Article electronically published on September 5, 2003

\title{
REFINING THE CONSTANT IN A MAXIMUM PRINCIPLE FOR THE BERGMAN SPACE
}

\author{
CHUNJIE WANG
}

(Communicated by Joseph A. Ball)

\begin{abstract}
Let $A^{2}(\mathbb{D})$ be the Bergman space over the open unit disk $\mathbb{D}$ in the complex plane. Korenblum conjectured that there is an absolute constant $c, 0<c<1$, such that whenever $|f(z)| \leq|g(z)|\left(f, g \in A^{2}(\mathbb{D})\right)$ in the annulus $c<|z|<1$, then $\|f\| \leq\|g\|$. In this note we give an example to show that $c<0.69472$.
\end{abstract}

Let $\mathbb{D}$ be the open unit disk in the complex plane $\mathbb{C}$. The Bergman space $A^{2}(\mathbb{D})$ consists of analytic functions $f$ in $\mathbb{D}$ such that

$$
\|f\|=\left[\int_{\mathbb{D}}|f(z)|^{2} d A(z)\right]^{\frac{1}{2}}<+\infty
$$

where

$$
d A(z)=\frac{1}{\pi} d x d y=\frac{1}{\pi} r d r d \theta, z=x+i y=r e^{i \theta}
$$

is the normalized Lebesgue area measure on $\mathbb{D}$. Korenblum $\mathbb{1}$ conjectured that there is an absolute constant $c, 0<c<1$, such that whenever $|f(z)| \leq|g(z)|$ in the annulus $c<|z|<1\left(f, g \in A^{2}(\mathbb{D})\right)$, then $\|f\| \leq\|g\|$.

W. K. Hayman [2] proved Korenblum's conjecture for $c=0.04$. Hinkkanen [3] improved Hayman's result that $c=0.157 \cdots$.

On the other hand, the example of $f(z)=\frac{1}{\sqrt{2}}, g(z)=z$ shows that $c \leq \frac{1}{\sqrt{2}}$. However, Martin (see [1]) gave the following example to show that $c=\frac{1}{\sqrt{2}}$ is not sharp.

Example. Let

$$
f(z)=\frac{1+(\sqrt{2}-1) z^{20}}{1+(\sqrt{2}-1) 2^{-10}}, \quad g(z)=\sqrt{2} z .
$$

Then $|f(z)| \leq|g(z)|$ for $\frac{1}{\sqrt{2}}<|z|<1$ but $\|f\|>\|g\|=1$.

In fact, an upper bound on $c$ can be found from Martin's example. Namely, if $f$ and $g$ are as in Martin's example, consider instead the pair $h$ and $g$, where $h=\frac{1}{\|f\|} f$. Then $\|h\|=\|g\|=1$ and $|h(z)| \leq|g(z)|$ in an annulus $c^{\prime}<|z|<1$. Using Mathematica and Lemma 1 below, we can easily obtain that $c^{\prime}=0.70450 \cdots<\frac{1}{\sqrt{2}}$.

Received by the editors October 28, 2002 and, in revised form, November 12, 2002.

2000 Mathematics Subject Classification. Primary 30C80, $30 \mathrm{H} 05$.

(C)2003 American Mathematical Society 
Lemma 1 (see [4]). If $f(z)=\sum_{k=0}^{+\infty} a_{k} z^{k} \in A^{2}(\mathbb{D})$, then

$$
\|f\|=\left(\sum_{k=0}^{+\infty} \frac{\left|a_{k}\right|^{2}}{k+1}\right)^{\frac{1}{2}} .
$$

Before stating our example, we recall that the singular inner functions are defined as

$$
S_{a}(z)=\exp \left(-a \frac{1+z}{1-z}\right),
$$

which play an important role in Bergman spaces [5], where $a$ is any positive constant. Our main result is the following.

Theorem. Let

$$
\begin{aligned}
& f(z)=e^{-a} S_{a}\left(z^{n}\right)=e^{-\frac{2 a}{1-z^{n}}}, \\
& g(z)=\frac{e^{-\frac{2 a}{1+c^{n}}}}{c} z,
\end{aligned}
$$

where $0<c<1, a=-\frac{1+c^{n}}{1-c^{n}} \log c>0, n \in \mathbb{N}$. Then $|f(z)| \leq|g(z)|$ in $c<|z|<1$. Moreover, when $n=14$ and $c=0.69472$, we have $\|f\|>\|g\|$.

Proof. It is easy to see that

$$
\varphi(r)=\max _{|z|=r}\left|\frac{f(z)}{g(z)}\right|=\frac{\max _{|z|=r}|f(z)|}{\frac{e^{-\frac{2 a}{1+c^{n}}}}{c} r}=\frac{e^{-\frac{2 a}{1+r^{r}}}}{e^{-a} r} .
$$

Hence, we have

$$
\varphi(c)=1, \varphi(1)=\lim _{r \rightarrow 1^{-}} \varphi(r)=1 .
$$

Since $\frac{f(z)}{g(z)}$ is analytic in $c \leq|z|<1$, the maximum modulus theorem implies that $|f(z)| \leq|g(z)|$ in $c<|z|<1$.

A direct calculation shows that the Taylor expansion of $f(z)$ at 0 is

$$
f(z)=e^{-2 a}\left[1-2 a z^{n}+2\left(a^{2}-a\right) z^{2 n}-\frac{4 a^{3}-12 a^{2}+6 a}{3} z^{3 n}+\cdots\right] .
$$

It follows from Lemma 1 that

$$
\begin{aligned}
& \int_{\mathbb{D}}|f(z)|^{2} d A(z)-\int_{\mathbb{D}}|g(z)|^{2} d A(z) \\
> & e^{-4 a}\left[1+\frac{4 a^{2}}{n+1}+\frac{4\left(a^{2}-a\right)^{2}}{2 n+1}+\frac{\left(4 a^{3}-12 a^{2}+6 a\right)^{2}}{9(3 n+1)}-\frac{e^{2 a}}{2}\right] \\
\triangleq & I(a) .
\end{aligned}
$$

Using Mathematica, we obtain that when $n=14$ and $c=0.69472$,

$$
e^{4 a} I(a)=0.0000214904>0 .
$$

So we have $\|f\|>\|g\|$.

Remark. It is likely that for all functions $f(z)$ and $g(z)$ (which depend on $n$ and $a>0$ ) defined in the theorem, $c=0.6947116 \cdots$ is the best one. 


\section{ACKNOWLEDGEMENT}

The author thanks the referee for several helpful suggestions, especially for pointing out that Martin's example can give an upper bound on the $c$ in Korenblum's theorem.

\section{REFERENCES}

[1] B. Korenblum, A maximum principle for the Bergman space, Publ. Mat. 35(1991), 479-486. MR 93j:30018

[2] W. K. Hayman, On a conjecture of Korenblum, Analysis (Munich) 19(1999), 195-205. MR 2000e:30041

[3] A. Hinkkanen, On a maximum principle in Bergman space, J. Anal. Math. 79(1999), 335-344. MR 2000m:30033

[4] H. Hedenmalm, Recent progress in the function theory of the Bergman space, pp. 35-50 in Holomorphic spaces, edited by S. Axler, J. E. McCarthy and D. Sarason, Mathematical Sciences Research Institute Publications 33, Cambridge University Press, 1998. MR 99e:46035

[5] H. Hedenmalm, B. Korenblum and K. Zhu, Theory of Bergman spaces, Springer-Verlag, New York, 2000. MR 2001c: 46043

School of Mathematical Sciences, Peking University, Beijing 100871, People's RePUBLIC OF CHINA

Current address: Department of Mathematics, Tianjin Polytechnic University, Tianjin, 300160, People's Republic of China

E-mail address: wcj498@eyou.com 